\title{
Anti-leukaemia effects of leonurine in vitro and in vivo
}

\author{
Qiang Zhuang ${ }^{1}$, Lina Ruan², Ting Jin ${ }^{3}$, Xiangkuo Zheng ${ }^{4}$ and Zhenlin Jin ${ }^{1}$ \\ ${ }^{1}$ Department of Hematology, The First Affiliated Hospital of Wenzhou Medical University, Wenzhou, China \\ ${ }^{2}$ Department of Animal Experimental Center, Wenzhou Medical University, Wenzhou, China \\ ${ }^{3}$ Operating Room, The First Affiliated Hospital of Wenzhou Medical University, Wenzhou, China \\ ${ }^{4}$ School of Laboratory Medicine and Life Sciences, Wenzhou Medical University, Wenzhou, China
}

\begin{abstract}
The present study was conducted to explore the anti-acute myeloid leukaemia (AML) effects of leonurine. HL-60 and U-937 cells were used to assess the antileukaemia effect of leonurine in vitro, and HL-60 and U-937 xenograft nude mice were used to evaluate its antitumour effect in vivo. Leonurine inhibited the proliferation of HL-60 and U-937 cells in a time- and dose-dependent manner. Moreover, leonurine therapy prevented the growth of tumours in both xenograft animal models. Leonurine could induce apoptosis in HL-60 and U-937 cells. The cytotoxic effects of leonurine on HL-60 and U-937 cells were associated with an increased ratio of Bax/Bcl-2, activation of caspase-3, caspase- 8 and caspase-9, and increased expression of cytochrome $c$ in the cytoplasm. Leonurine inhibited activation of the PI3K/Akt pathway in HL-60 and U-937 cells by lowering the phosphorylation levels of PI3K and Akt. Our results indicate that leonurine is a potential anti-AML agent, and this activity may be associated with its repression of the phosphorylation of PI3K and Akt.
\end{abstract}

Key words: Leonurine - Acute myeloid leukaemia - PI3K - Akt - HL-60 - U-937

\section{Introduction}

Acute myeloid leukaemia (AML) starts inside the bone marrow and is one of the most common acute leukaemia in adults and among the most lethal. In the United States, the annual incidence of AML is 19,000 new cases, and the incidence of AML-associated deaths is 10,000 (Kadia et al. 2016). At present, radiotherapy and chemotherapy (daunorubicin, cytarabine and decitabine) are available to prevent the development of AML in the clinic. However, because of suboptimal response, resistance, morbidity from drug toxicities and disease relapse, a considerable proportion of patients do not achieve complete remission (Kuykendall et al. 2018). AML remains a disease with poor prognosis and high mortality. Only one-fifth of patients suffering from AML during the period from 1997 to 2013 were alive on 1 January 2014, and

Correspondence to: Zhenlin Jin, Department of Hematology, The First Affiliated Hospital of Wenzhou Medical University, Nanbaixiang Street, Ouhai District, Wenzhou, 325000, Zhejiang Province, China E-mail: jinzhenlin1999@163.com one-third of these patients had been diagnosed within the past 3 years (Juliusson et al. 2017). Therefore, efforts to develop novel, safe and effective treatments for AML are necessary.

Natural products or active compounds from natural plants have been extensively studied for their good antitumour activity in many types of malignant tumours, and some of them (paclitaxel and vincristine) have been widely used to treat cancer in patients (Thomas et al. 2006; Williamson et al. 2015). The advantages of using herbal medicines for tumour prevention in the clinic include fewer side effects, lower cost and more therapeutic benefits (Liu et al. 2014; Qi et al. 2015). Herba leonuri is a famous Chinese herbal medicine that has been extensively used to treat various gynaecological diseases (Zhu et al. 2018). Leonurine, which is extracted from Herba leonuri, possesses great activities to be beneficial for cardiovascular diseases, rheumatoid arthritis and endometritis (Li et al. 2017; Wu et al. 2018; Xu et al. 2018). Moreover, leonurine has been proven to inhibit tumour cell proliferation and to induce cell cycle arrest and apoptosis in the H292 cancer cell line (Mao et al. 2015). In the present study, we aimed to determine whether leonurine

(C) The Authors 2021. This is an open access article under the terms of the Creative Commons Attribution-NonCommercial 4.0 International License (https://creativecommons.org/licenses/by-nc/4.0/), which permits non-commercial use, distribution, and reproduction in any medium, provided the original work is properly cited. 
inhibits AML development both in vivo and in vitro and to determine the mechanism(s) involved.

\section{Materials and Methods}

\section{Cell lines and culture}

Human AML cell lines HL-60 and U-937 (ATCC ${ }^{\circledR}$ CRL$\left.1593.2^{\mathrm{TM}}\right)$ were obtained from American Type Culture Collection (Virginia, USA). Human peripheral blood samples were collected from healthy adult individuals based on approval by the Committee on Human Subjects in Research of the First Affiliated Hospital of Wenzhou Medical University and following written informed consent by the donors. Peripheral blood mononuclear cells (PBMCs) were collected using a Ficoll-Paque gradient as described previously (Riedhammer et al. 2016). All cells were grown in RPMI-1640 medium supplemented with 10\% FBS, L-glutamine (300 mg/l), $100 \mathrm{IU} / \mathrm{ml}$ penicillin, and $100 \mu \mathrm{g} / \mathrm{ml}$ streptomycin and placed in humidified air at $37^{\circ} \mathrm{C}$ in a $5 \% \mathrm{CO}_{2}$ atmosphere.

\section{Proliferation inhibition assay}

HL-60, U-937 and PBMCs were seeded on 96-well plates at a density of $1 \times 10^{4}$ cells/well $(200 \mu \mathrm{l} /$ well $)$ and incubated for 24 and $48 \mathrm{~h}$ at $37^{\circ} \mathrm{C}$ with different concentrations of leonurine (purity beyond 98\%, Sigma, St. Louis, MO, USA). Afterwards, the medium was replaced with fresh medium containing $5 \mathrm{mg} / \mathrm{ml}$ MTT for $4 \mathrm{~h}$. Absorbance was measured at $570 \mathrm{~nm}$ by using a microplate reader (Thermo Fisher Scientific, Waltham, MA, USA). Three replications of each experiment were performed, and fifty percent of the inhibitory concentration (IC50) value was calculated by GraphPad Prism 5 software (GraphPad Software, San Diego, CA, USA). Moreover, a similar experiment was conducted for $48 \mathrm{~h}$ in HL-60 or U-937 cells that received different concentrations of methotrexate (MTX, purity beyond 98\%, Sigma, St. Louis, MO, USA) or vincristine (purity beyond 98\%, Sigma, St. Louis, MO, USA), and viability was measured in the same way.

\section{Evaluation of apoptosis}

HL-60 and U-937 cells were seeded on 6-well plates at a density of $1 \times 10^{5}$ cells/well ( $2 \mathrm{ml} /$ well $)$ and treated with leonurine at concentrations of 2,5 , and $10 \mu \mathrm{M}$ for $48 \mathrm{~h}$. Afterwards, apoptosis was evaluated by using an annexin V-PI staining kit (BD Biosciences, San Jose, CA, USA) according to the manufacturer's protocol. After $48 \mathrm{~h}$ of incubation, the cells were centrifuged $(500 \times g, 10 \mathrm{~min})$ and stained with $1 \mu \mathrm{l} 20 \mu \mathrm{g} /$ $\mathrm{ml}$ annexin $\mathrm{V}$-FITC and $2 \mu \mathrm{l} 50 \mu \mathrm{g} / \mathrm{ml} \mathrm{PI}$ for $15 \mathrm{~min}$ at room temperature in the dark. The detection of apoptotic death was performed in a flow cytometer (Becton-Dickinson, USA), and the data were obtained by using CellQuest software.

\section{Measurement of caspase activities}

HL-60 and U-937 cells were seeded on 6-well plates at a density of $5 \times 10^{5}$ cells/well ( $2 \mathrm{ml} /$ well $)$ and treated with leonurine at concentrations of 2, 5, and $10 \mu \mathrm{M}$ for $48 \mathrm{~h}$. Caspase-3, caspase- 8 , and caspase- 9 activities were measured using commercial colorimetric assay kits (catalogue numbers: K106-100; K113-100; K119-100; R\&D Systems, Minneapolis, MN, USA). Briefly, the control cells and leonurine-treated cells were lysed in the supplied lysis buffer. The supernatants were collected and incubated with supplied reaction buffer containing dithiothreitol and DEAD-pNA, IETD-pNA, or LEHD-pNA as substrates at $37^{\circ} \mathrm{C}$ for $2 \mathrm{~h}$ in the dark. The reactions were measured by changes in absorbance at $405 \mathrm{~nm}$ using a microplate reader (Thermo Fisher Scientific, Waltham, MA).

\section{Western blot analysis}

Both HL-60 and U-937 cells were treated with leonurine (2, 5, and $10 \mu \mathrm{M}$ ) for $48 \mathrm{~h}$. All cells were harvested and lysed in icecold radioimmunoprecipitation lysis buffer. The protein concentration was determined using a quantitative assay kit based on Bradford method (cat.no.: 5000001; Bio-Rad, Richmond, CA, USA). Equal amounts of lysate protein were subjected to SDS-PAGE. The proteins were electrotransferred to polyvinylidene fluoride membranes, and the blots were incubated with primary antibodies against Bcl-2 (cat.no.: sc-7382; Santa Cruz, CA, USA), Bax (cat.no.: sc-7480; Santa Cruz, CA, USA), cytochrome $c$ (cat.no.: sc-13156; cyt-c, Santa Cruz, CA, USA), phosphorylated (p)-PI3K (cat.no.: 13857; Cell Signalling Technology, Beverly, MA, USA), PI3K (cat.no.: abs111482; Absin; Shanghai, China), p-Akt (cat.no.: 3152005A; R\&D Systems, USA) and Akt (cat.no.: 3427; Biovision, CA, USA) overnight at $4^{\circ} \mathrm{C}$. After that, the membrane was washed and incubated with horseradish peroxidase-conjugated antibody (cat.no.: A1092; A0208; and A0216; Beyotime, Haimen, China) for $2 \mathrm{~h}$ at room temperature. Finally, the protein bands were stained with ECL reagent (GE Healthcare, Danvers, MA, USA). The relative intensity of bands was quantified using ImageJ Software (National Institutes of Health, Bethesda, $\mathrm{MD}$, USA), and data were normalized to the corresponding loading control $\beta$-actin (incubation with membranes with a dilution of 1:2000, cat.no: sc-47778; Santa Cruz, CA, USA) and expressed as the fold change of the control.

\section{Xenograft animal model}

The animal study was approved by the Institutional Animal Care and Use Committee (IACUC) of the First Affiliated Hospital of Wenzhou Medical University (approved number: 
20171104, Wenzhou, China). Nude mice (5 weeks old, male) were obtained from the Model Animal Research Center of Nanjing University (Nanjing, China). Mice were maintained under standardized, sterilized conditions $\left(24 \pm 2^{\circ} \mathrm{C}, 60-70 \%\right.$ relative humidity, $12 \mathrm{~h}$ dark/light cycle) in a specific pathogenfree laboratory and were fed regular nude mouse chow. All operations in the experiment were conducted under the guidelines of laboratory animal use and care of the European Community (EEC Directive of 1986; 86/609/EEC). To induce a xenografted tumour model, mice were injected subcutaneously with HL-60 cells $\left(1 \times 10^{7} / 0.2 \mathrm{ml} /\right.$ each mouse) or U-937 cells $\left(1 \times 10^{7} / 0.2 \mathrm{ml} /\right.$ each mouse) into the right flank (day 0$)$. When the average tumour volume reached $100 \mathrm{~mm}^{3}$, mice were randomly assigned to four groups with 6 mice per group: the control (vehicle) group and three groups of leonurine $(15,30$, and $60 \mathrm{mg} / \mathrm{kg}$, intragastric administration). Tumour size was measured every four days. Tumour volumes were calculated according to the formula (width ${ }^{2} \times$ length) $/ 2$. All animals were sacrificed immediately after 20 days of drug exposure, and tumours were removed and weighed.

\section{TUNEL assay}

To evaluate apoptosis in tumour tissue sections, TUNEL labelling was performed using an in situ cell death detection kit (cat.no.: C1086; Beyotime, Haimen, China). Briefly, tissue sections of formalin-fixed, paraffin-embedded specimens were dewaxed in xylene and rehydrated in a graded series of ethanol. Afterwards, DNA fragmentation was detected according to the manufacturer's instructions. For quantification, three different fields were counted under confocal laser microscopy (Leica Microsystems, Heerbrugg, Switzerland), and at least 100 cells were enumerated in each field.

\section{Statistical analysis}

Data are shown as the mean \pm standard deviation (SD). Statistical software SPSS 15.0 (SPSS Inc., Chicago, IL, USA) was used to analyse data by one-way analysis of variance (ANOVA) followed by LSD test. Significant differences were defined as $p<0.05$.

A
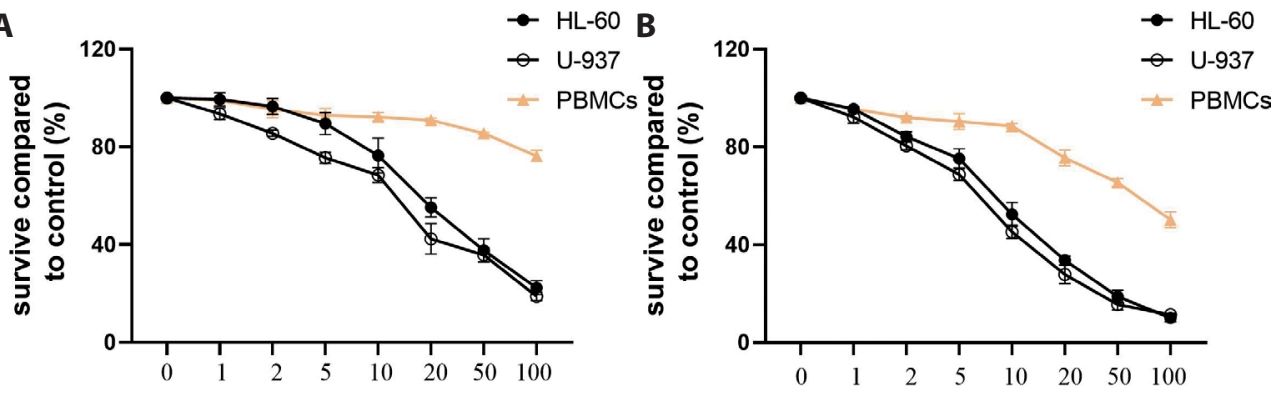

C

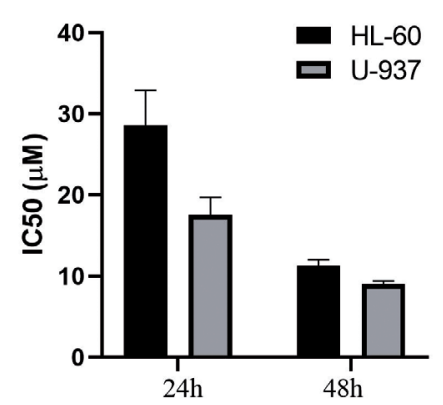

E

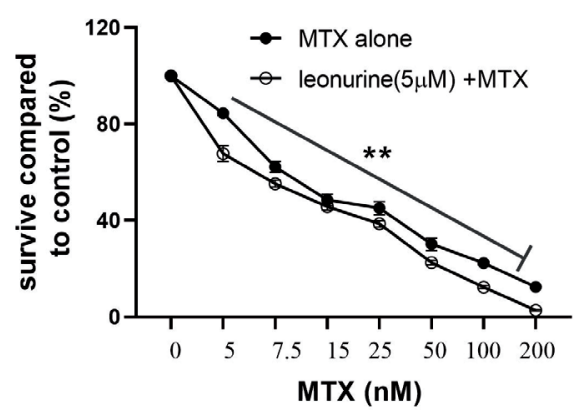

\section{D}

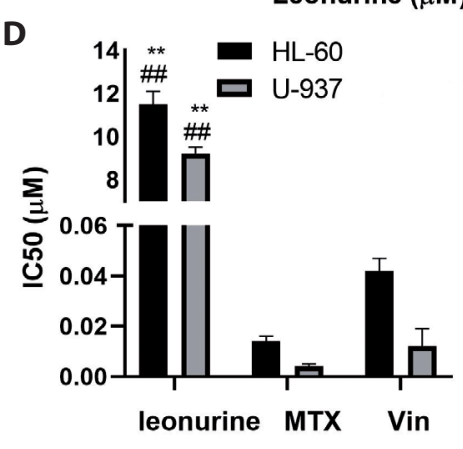

$\mathbf{F}$

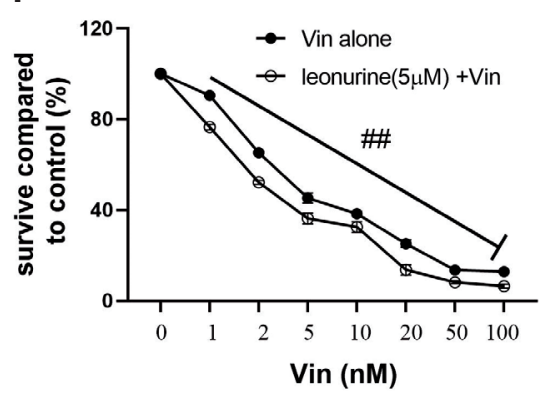

Figure 1. Antiproliferative effects of leonurine on HL-60 and U-937 cells. Cell viability of HL-60, U-937 and PBMCs treated with leonurine $(1,2$, $5,10,20,50$ and $100 \mu \mathrm{M})$ for $24 \mathrm{~h}(\mathbf{A})$ and $48 \mathrm{~h}(\mathbf{B})$. C. IC50 values of leonurine on HL-60 and U-937 cells with incubation times 24 and 48 h. D. Effect of leonurine, MTX and vincristine on HL-60 and U-937 cells with $48 \mathrm{~h}$ incubation time. ${ }^{* *} p<$ 0.01 compared with the MTX group, ${ }^{\# \#} p<0.01$ compared with the Vin group. Cell viability of HL-60 cells treated with $1,2,5,10,20,50$ and $100 \mu \mathrm{M}$ MTX (E) and vincristine (F) for $48 \mathrm{~h}$ in the presence of $5 \mu \mathrm{M}$ leonurine. ${ }^{* *} p<0.01$ compared with the leonurine+MTX group, \#\# $p<0.01$ compared with the leonurine+Vin group. Data are presented as the mean \pm SD. Three independent experiments were performed in duplicate. IC50, 50\% inhibitory concentration; PBMCs, peripheral blood mononuclear cells; MTX, methotrexate; Vin, vincristine. 


\section{Results}

\section{Leonurine inhibits proliferation of HL-60 and U-937 cells}

AML cell lines and PBMCs were treated with leonurine for $24 \mathrm{~h}$ (Fig. 1A) and $48 \mathrm{~h}$ (Fig. 1B), and the viability of cells was measured. Leonurine showed a good inhibitory effect on the viability of HL-60 and U-937 cells. The IC50 values of leonurine for inhibiting HL-60 cell viability were $28.6 \mu \mathrm{M}(24 \mathrm{~h})$ and $11.3 \mu \mathrm{M}(48 \mathrm{~h})$, and the IC50 values for inhibiting U-937 cell viability were $17.5 \mu \mathrm{M}(24 \mathrm{~h})$ and $9.0 \mu \mathrm{M}$ (48 h) (Fig. 1C).
The IC50 values of leonurine for inhibiting HL-60/U-937 cell viability were significantly higher than those of MTX (14.0 nM/4.1 nM) and vincristine (42.0 nM/12.2 nM) (Fig. 1D). To study further the cytotoxic selectivity of leonurine, we investigated the effects of leonurine on the viability of PBMCs. The data reflected in Figure $1 \mathrm{~A}$ and $\mathrm{B}$ revealed that PBMCs were not sensitive to leonurine even with high and anti-AML concentrations. As shown in Figure 1E, treatment of HL-60 cells with MTX together with leonurine showed more powerful inhibitory effects on viability than MTX alone $(p<0.01)$. Likewise, as seen in Figure 1F, we observed syner-

\section{Leonurine $(\mu \mathrm{M})$}

A

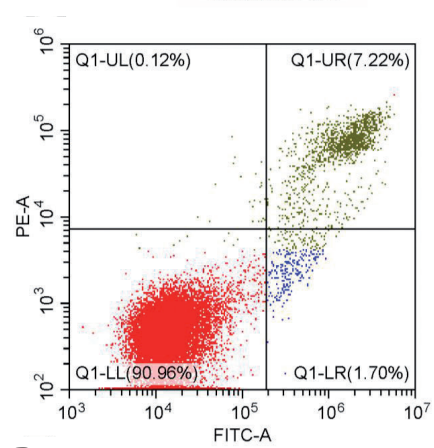

B
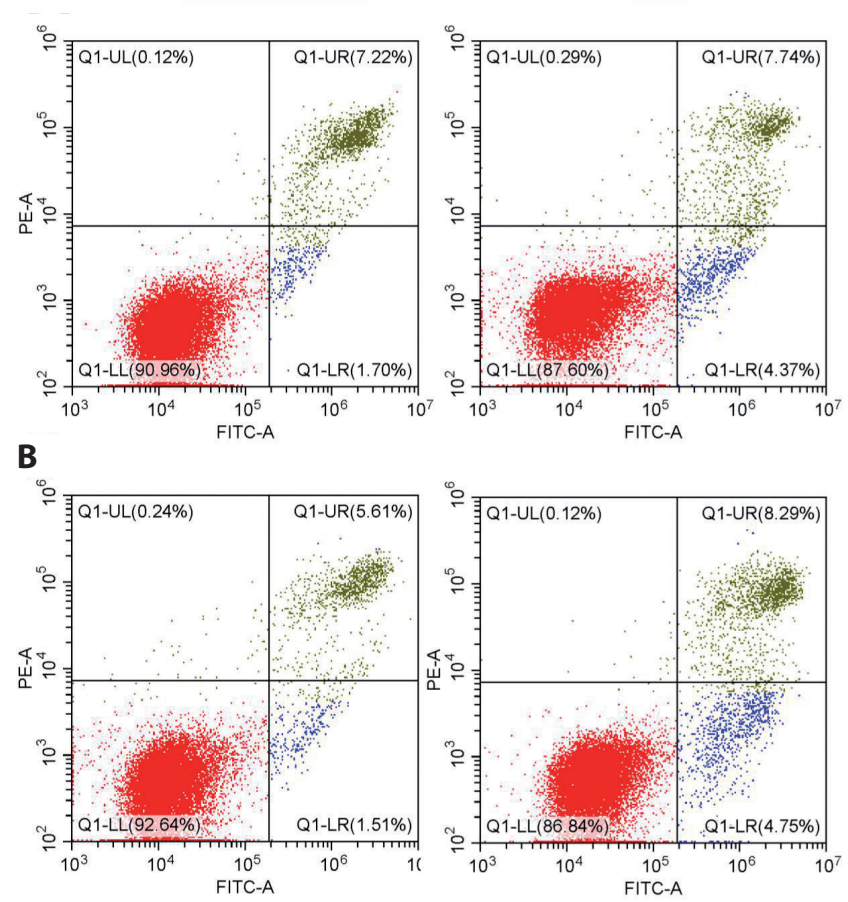

2

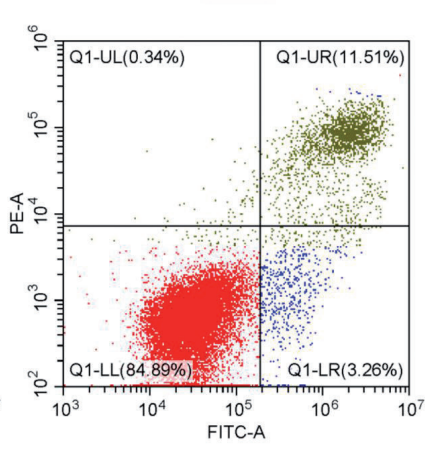

5
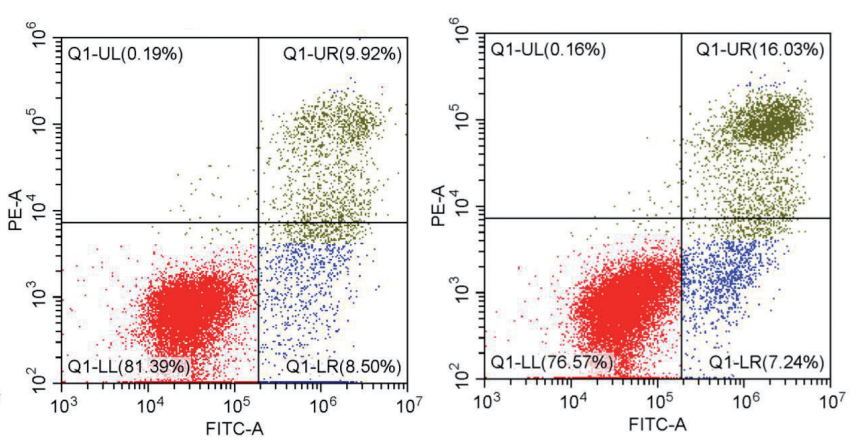

\section{C}

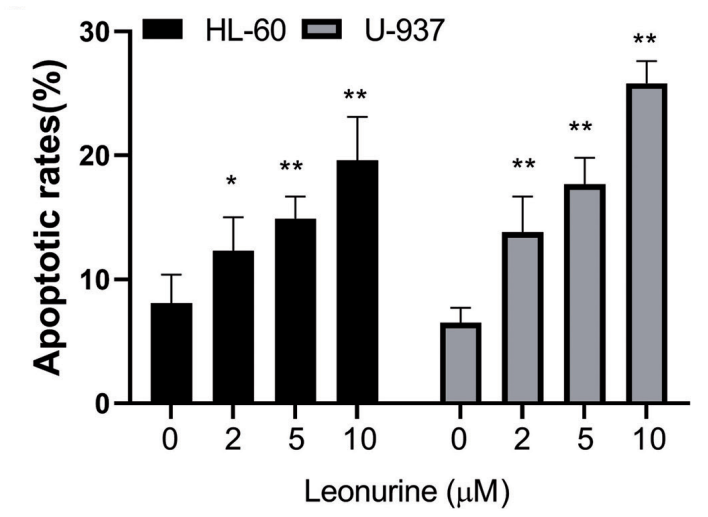

Figure 2. Apoptotic death of HL-60 and U-937 cells induced by leonurine. HL-60 cells (A) and U-937 cells (B) were treated with leonurine for $48 \mathrm{~h}$ and analysed by flow cytometry. C. Percentages of apoptotic cells; the data was calculated based on the sum of early aopoptotic cells (blue plots) and late apoptotic cells (green plots) numbers. Data are presented as the mean \pm SD. ${ }^{*} p<0.05$ and ${ }^{\star *} p<0.01$ compared with the Control group (in the absence of leonurine). Three independent experiments were performed in duplicate. For color Figure, see on-line version of the manuscript. 

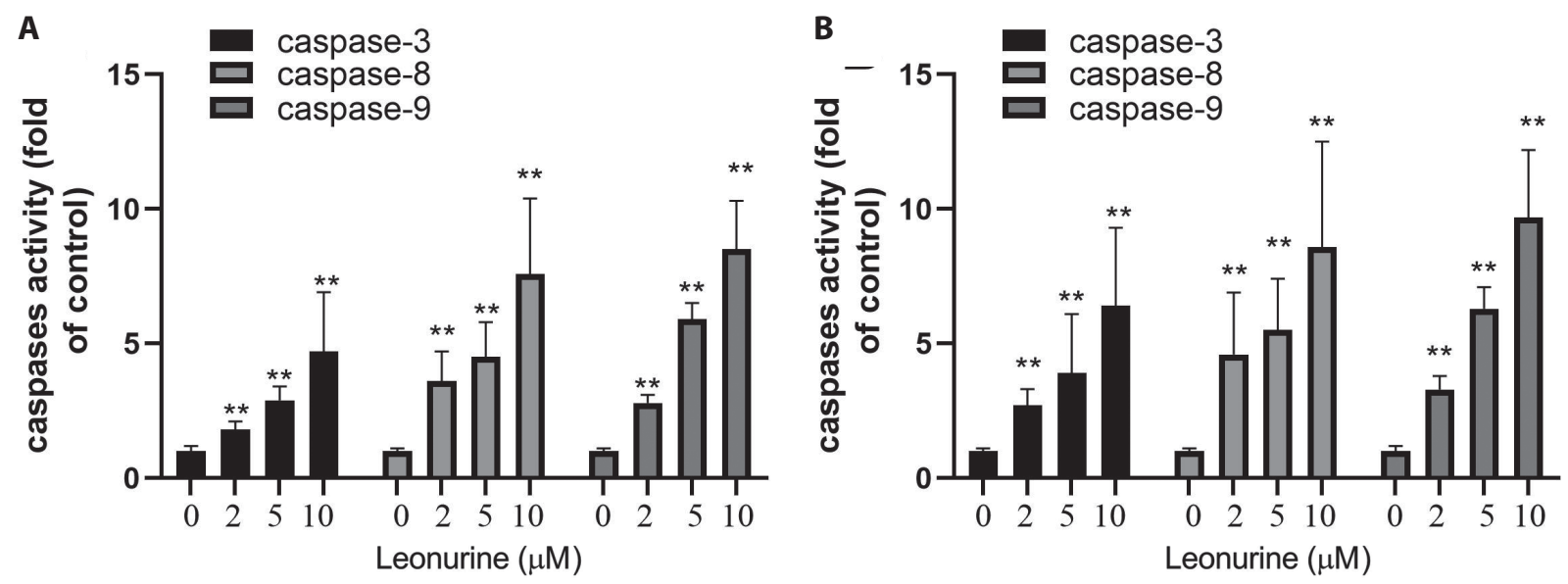

Figure 3. Activation of caspase-3, caspase- 8 and caspase-9 in HL-60 (A) and U-937 (B) cells induced by leonurine. Data are presented as the mean $\pm \mathrm{SD}$. ${ }^{*} p<0.01$ compared with the group without leonurine treatment. Three independent experiments were performed in duplicate.

gistic antiproliferative action when vincristine and leonurine were used together, which showed more inhibitory effects on viability of HL-60 compared with that of control group $(p<0.01)$. Moreover, we compared the combined group (contained reduced concentration of MTX or vincristine) with MTX alone/vincristine alone group. It could be found the combined treatment almost showed the same inhibitory effects $(p>0.05)$ on the HL-60 viability to those of higher concentration of MTX/vincristine.

\section{Leonurine induces apoptosis in HL-60 and U-937 cells}

The percentage of apoptotic HL-60 cells treated with leonurine was determined (Fig. 2A and C). Briefly, after treatment for $48 \mathrm{~h}, \mathrm{HL}-60$ cells exhibited 1.5-fold, 1.8-fold and 2.4-fold increases in apoptosis in response to 2, 5, and $10 \mu \mathrm{M}$ leonurine, respectively. Moreover, we also found that U-937 cells showed 2.1-fold, 2.7-fold and 4.0-fold increases in apoptotic death in response to 2,5 , and $10 \mu \mathrm{M}$ leonurine, respectively (Fig. 2B and C). Our data suggest that leonurine promotes apoptotic death in both HL-60 and U-937 cells.

\section{Leonurine induces activation of caspases}

The upregulation of caspase cascades is an important event during the activation of extrinsic and intrinsic apoptosis signalling pathways. Therefore, we further investigated the effects of leonurine treatment on caspase activity in HL-60 and U-937 cells. HL-60 cells exhibited 1.8-fold, 2.9 -fold and 4.7-fold increases in the activation of caspase-3; 3.6-fold, 4.5-fold and 7.6-fold increases in the activation of caspase-8; and 2.8-fold, 5.9-fold and 8.5-fold increases in the activation of caspase- 9 in response to 2,5 , and $10 \mu \mathrm{M}$ leonurine, respectively $(p<0.01)$ (Fig. 3A). Moreover, we observed a similar change in caspase activity in U-937 cells treated with leonurine (Fig. 3B).

\section{Leonurine regulates apoptotic and prosurvival protein expression}

We further studied the influences of leonurine on apoptosisrelated protein levels in AML cells. As shown in Figure 4A, leonurine-treated HL- 60 cells exhibited increased levels of Bax and decreased levels of Bcl-2 compared with control cells $(p<$ $0.01)$. Moreover, we found that leonurine treatment induced an increased expression of cyt-c in HL-60 cells. Similarly, we also noted that U-937 cells that received leonurine treatment exhibited increased levels of Bax and cyt-c and decreased levels of Bcl-2 compared with control cells $(p<0.01)$ (Fig. 4B).

Leonurine blocks activation of the PI3K-Akt signalling pathway in HL-60 and U-937 cells

To elucidate the underlying molecular mechanism of leonurine-induced apoptosis, the levels of the PI3K/Akt signalling pathway were examined using Western blotting. Leonurine-treated HL-60 cells exhibited decreased levels of p-PI3K and p-Akt compared with control cells $(p<0.01)$ (Fig. 5A). Moreover, we found that leonurine had almost no effects on the total expression of PI3K and Akt. Similarly, we also noted that U-937 cells that received leonurine treatment exhibited a reduction in the levels of $\mathrm{p}-\mathrm{PI} 3 \mathrm{~K}$ and $\mathrm{p}$-Akt $(p<0.01)$ and no change in the total expression of PI3K and Akt (Fig. 5B). All these findings suggest that leonurine may exert its inhibitory effects on the proliferation of HL-60 and U-937 cells via the PI3K/Akt pathway. 


\section{Leonurine $(\mu \mathrm{M})$}

\begin{tabular}{llll}
\hline 0 & 2 & 5 & 10
\end{tabular}

$\mathrm{Bcl}-2$

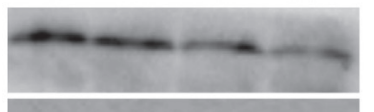

Bax

Cyt-c

$\beta$-actin

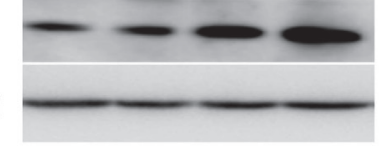

Leonurine $(\mu \mathrm{M})$

B

\begin{tabular}{llll}
\hline 0 & 2 & 5 & 10
\end{tabular}

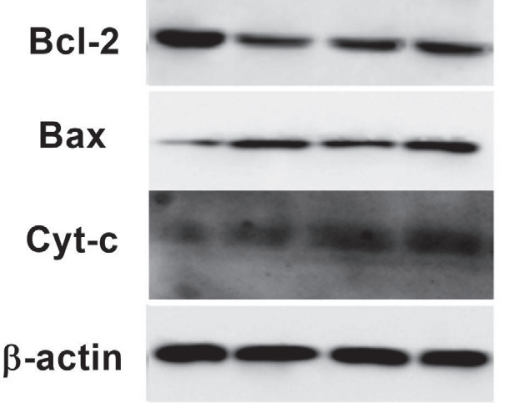

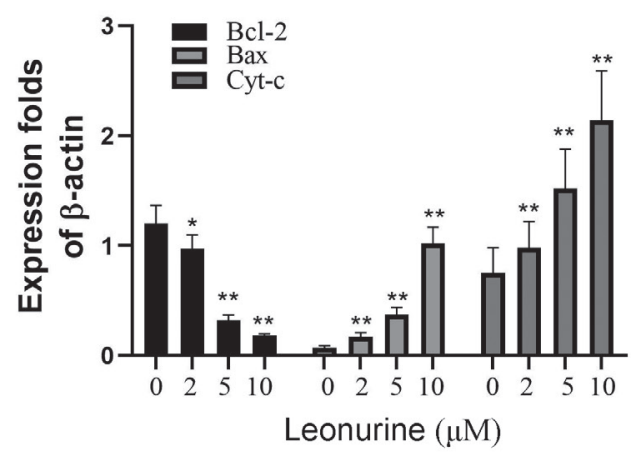

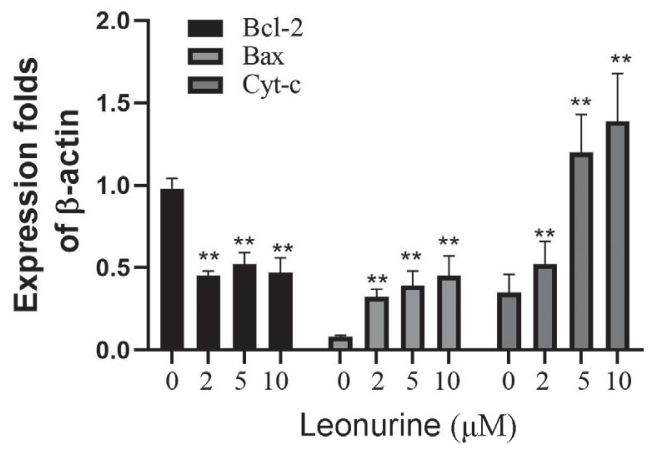

Figure 4. Leonurine regulates apoptotic proteins in HL-60 and U-937 cells. HL-60 (A) and U-937 (B) cells were treated with leonurine for $48 \mathrm{~h}$, and intracellular proteins was detected by Western blot analysis. Data are presented as the mean \pm SD. Three independent experiments were performed in duplicate. ${ }^{* *} p<0.01$ compared with the group in the absence of leonurine. Cyt-c, cytochrome $c$.

\section{Leonurine inhibits tumour growth in HL-60 and U-937} xenografted nude mice

To evaluate further the anti-AML activity of leonurine in vivo, we engrafted HL-60 and U-937 cells into nude mice and treated the animals daily with oral administration of vehicle or leonurine $(15-60 \mathrm{mg} / \mathrm{kg})$. The data shown in Figure 6A and $\mathrm{C}$ indicated a significant decrease in the tumour volume and tumour weight of leonurine-treated HL-60 xenograft animals $(p<0.01)$. Moreover, as seen in Figure 6B and C, we observed a similar decrease in the tumour volume and weight in U-937 xenografted mice treated with leonurine ( $p<$ $0.01)$. We speculated that the decrease in tumour volume was due to the enhanced apoptotic death induced by leonurine. Therefore, we continued to detect apoptosis of tumour tissue by using TUNEL staining. In both animal models with xenografted tumours, treatment with leonurine induced increased apoptosis in the tumour tissue, as examined by TUNEL staining $(p<0.01)$ (Fig. 7A and B).

\section{Discussion}

In a previous report, leonurine was demonstrated to exert antiproliferative activity in the $\mathrm{H} 292$ cancer cell line (Mao et al. 2015). We suspect that leonurine may have some inhibi- tory effects on AML. No report is available for leonurine's effect on AML. Therefore, the present study was designed to determine the effect of leonurine on AML development both in vivo and in vitro. Our results showed that leonurine inhibited the proliferation of AML cell lines (HL-60 and U-937) in a time- and dose-dependent manner, with IC50 values ranging from $11.3 \mu \mathrm{M}$ to $28.6 \mu \mathrm{M}$ for HL-60 cells and 9.0 $\mu \mathrm{M}$ to $17.5 \mu \mathrm{M}$ for U-937 cells. These data were similar to a previous report that leonurine at concentrations from $10 \mu \mathrm{M}$ to $50 \mu \mathrm{M}$ inhibited the proliferation of $\mathrm{H} 292$ cells in vitro (Mao et al. 2015). Based on suggestions from a study (Mans et al. 2000), natural active medicine with an IC50 value less than or equal to $50 \mu \mathrm{g} / \mathrm{ml}$ would be worth submitting to a more comprehensive disease-oriented screen of the U.S. National Cancer Institute. In our study, the IC50 value of leonurine for treating AML cell lines supported that leonurine exerted significant antileukaemia activity. Although the IC50 value of leonurine was significantly higher than those of MTX and vincristine, it is interesting to see that treatment of AML cells with MTX or vincristine together with leonurine exhibited more powerful inhibition of the viability of HL- 60 cells than treatment with MTX or vincristine alone. Our data suggest that leonurine and MTX/vincristine could synergistically inhibit the survival of HL-60 cells; however, we do not know the specific synergistic mechanism involved in the action. Moreover, our data showed that PBMCs were not sensitive 
to leonurine treatment even with a high anti-AML concentration at $10 \mu \mathrm{M}$, which is a concentration much higher than that exposure in vivo ( $\mathrm{Li}$ et al. 2013). A previous study (Hirano et al. 1996) found that many anti-AML cytotoxic drugs (such as etoposide, doxorubicin, vincristine and MTX) showed very poor selectivity between AML cells and normal cells, as reflected by their IC50 values. Some of these drugs exhibit significant cytotoxic effects on PBMCs with low IC50 values (vincristine: $3.2 \mathrm{nM}$ and MTX: $4.8 \mathrm{nM}$ ). Therefore, leonurine may have better safety than conventional cytotoxic drugs when treating AML. In an in vivo study, we further evaluated the anti-AML activity of leonurine by measuring tumour size in mice that received leonurine treatment. The results revealed that leonurine could reduce the size of tumours in HL-60 and U-937 xenograft tumour models, and the animals didn't show any observed side effects (such as vomiting, lethargy, listlessness, diarrhea and others) during treatment. Our data indicate that leonurine showed good anti-AML activity as well as security.

Apoptosis, known as programmed cell death, is involved in pharmacological actions by most anticancer drugs. Therefore, apoptotic death was analysed by Annexin V-PI staining in vitro and TUNEL staining in vivo to elucidate the type of cell death induced by leonurine. We demonstrated that leonurine could increase the numbers of apoptotic cells both in vitro and in vivo. Intrinsic (mitochondria) and extrinsic (death receptor)-mediated apoptotic pathways are the main forces that execute and drive apoptotic death in cells (Ma et al. 2016; Yu et al. 2017). Briefly, in the intrinsic apoptotic pathway, B-cell lymphoma 2 family members, such as Bcl-2 and $B a x$, are the main upstream molecules in response to apoptosis signals. Activation of apoptotic proteins results in mitochondrial dysfunction, which promotes the release of some characteristic mitochondrial factors (such as cyt-c) from the mitochondria to the cytoplasm and induces caspase- 9 and caspase- 3 activation, all of which finally trigger the intrinsic apoptotic pathway (Ma et al. 2016; Yu et al. 2017). In the extrinsic apoptotic pathway, the death receptor stimulates expressed death ligands and activates downstream molecules (such as caspase- 8 and caspase-3) to initiate cell death. In the present study, leonurine increased the expression of bax and reduced the expression of bcl-2, indicating apoptosis in leonurine-treated AML cells. Moreover, we found that leonurine resulted in an increase in the expression
A

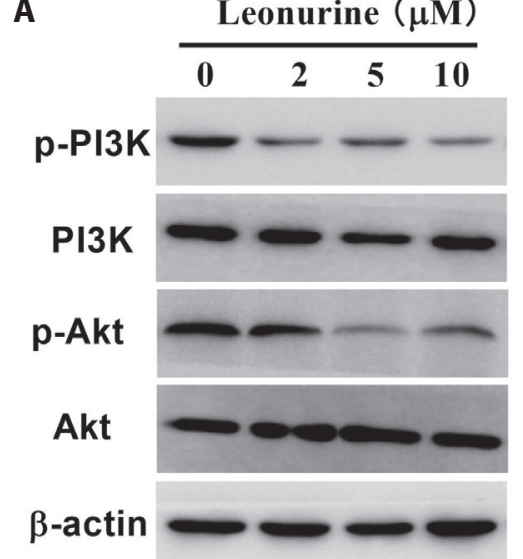

B

Leonurine $(\mu \mathrm{M})$

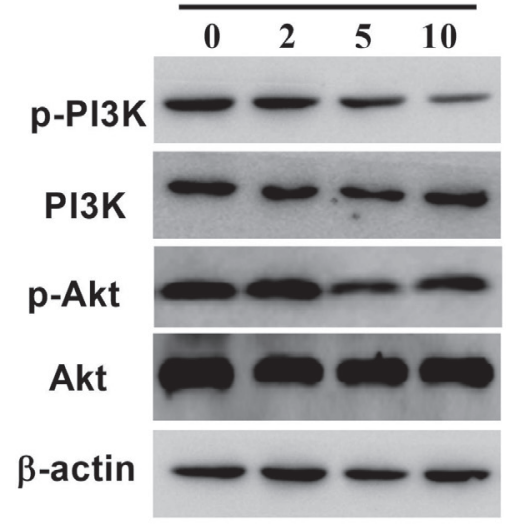

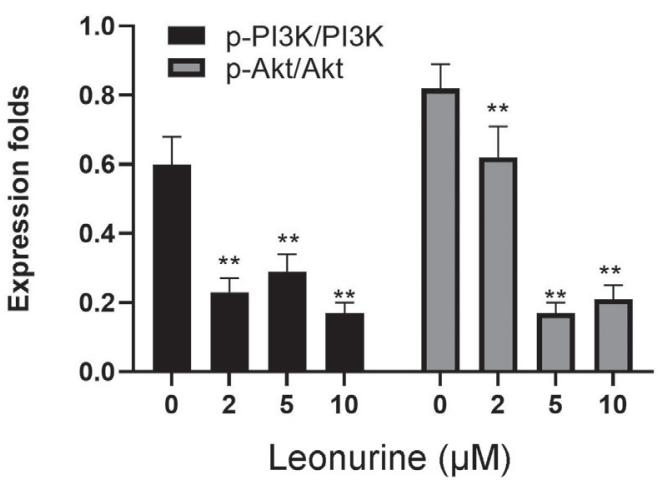

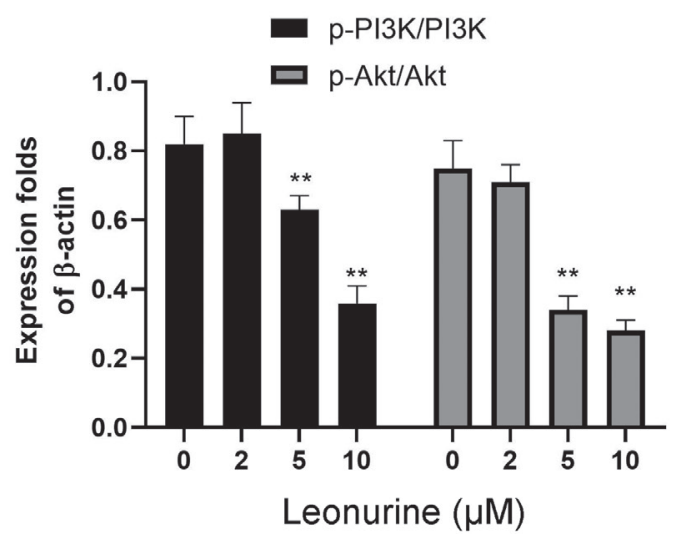

Figure 5. Leonurine blocks the activation of PI3K/Akt in HL-60 and U-937 cells. HL-60 (A) and U-937 (B) cells were treated with leonurine for $48 \mathrm{~h}$, and intracellular p-PI3K, PI3K, p-Akt and Akt were detected by Western blot analysis. Data are presented as the mean \pm SD. Three independent experiments were performed in duplicate. ${ }^{* *} p<0.01$ compared with the group without leonurine treatment. 


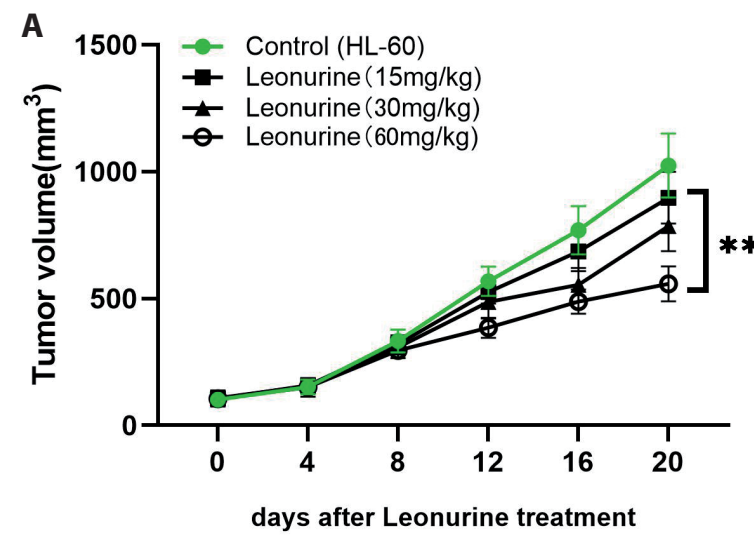

days after Leonurine treatment

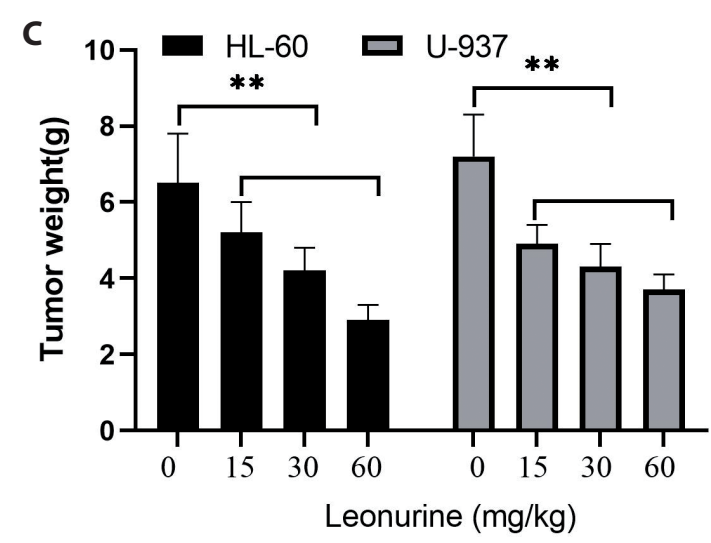

of cytoplasmic cytochrome-c and caused an enhancement in the activity of caspase- 3 , caspase- 8 and caspase- 9 . Our results indicate that leonurine-mediated apoptosis in AML cells occurs via activation of intrinsic and extrinsic apoptotic pathways. Interestingly, several published reports have shown that leonurine possesses similar antiapoptotic activity. In in vivo studies (Shi et al. 2011; Xu et al. 2018), leonurine exerts cardiac protection in acute myocardial infarction rats by its anti-apoptotic effects. Furthermore, leonurine exhibits neuroprotective effects on 6-hydroxydopamine-induced behavioural deficits in rats via its antiapoptotic activity. In in vitro studies (Xin et al. 2009; Liu et al. 2013; Hu PF et al. 2019), leonurine has been proven to exert antiapoptotic effects in IL- $1 \beta$-treated chondrocytes, doxorubicin-treated cardiac muscle cells, and hypoxia-treated cardiac myocytes. Based on these reports and our results, it seems that leonurine shows "bidirectional actions", and the choice of proapoptotic or antiapoptotic effects of leonurine is determined by the type of disease and the status and type of cells. Apoptosis is triggered in intrinsic and/or extrinsic ways, and each pathway is regulated by many different signalling molecules. We believe that leonurine is an active substance with multiple targets that has not been revealed.

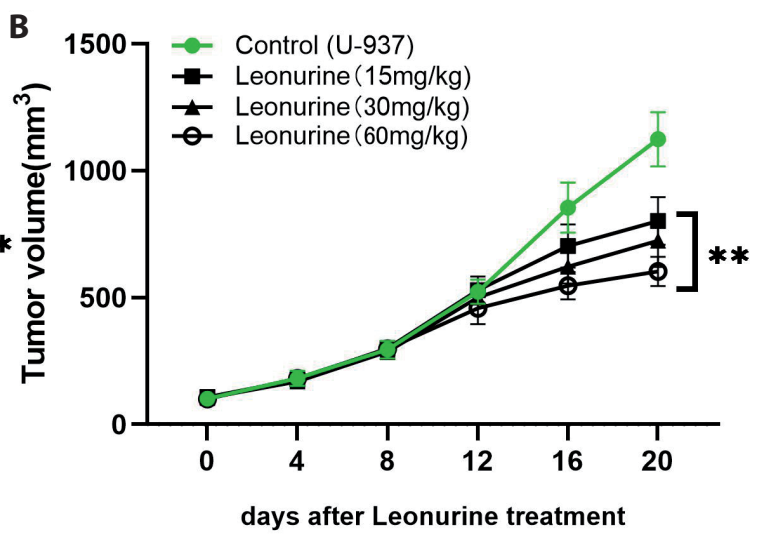

Figure 6. Leonurine inhibits tumour growth in HL-60 and U-937 xenografted nude mice. BALB/c nude mice were injected with HL-60 cells (A) or U-937 cells (B) and then divided into a control group (received vehicle - PBS) and three groups treated with leonurine in concentrations 15 , 30 and $60 \mu \mathrm{M}$ for 20 days. C. Tumour weight was measured in all sacrificed mice. Data are presented as the mean \pm SD; $n=6 .{ }^{*} p<0.01$ compared with the Control group in the absence of leonurine.

PI3K/Akt is an intracellular signalling pathway of great importance in multiple cellular physiological processes, including cellular quiescence, proliferation, apoptosis and others (Bertacchini et al. 2015; Xie et al. 2019). Generally, the PI3K cascade is activated by many types of cellular stimuli. PI3K activation further activates the phosphorylation process of Akt, which localizes to the cellular membrane. Activated Akt fulfills various biological functions. The role of PI3K/Akt in the development of AML has been demonstrated. Clinical observations have demonstrated that most ALM patients exhibit higher activation of PI3K/Akt and are associated with decreased overall survival (Xu et al. 2003; Tamburini et al. 2007). Moreover, the efficacy of selective PI3K/Akt inhibitors has been investigated in AML cell lines. Briefly, a specific PI3K isoform inhibitor decreased rRNA synthesis and cell proliferation of AML cells (Nguyen et al. 2014). Consistent with these results, preclinical evaluation of the Akt inhibitor MK2206 demonstrated that MK2206 sensitizes AML primary cells to gemtuzumab ozogamicin therapy (Rosen et al. 2013). It has been recently reported that leonurine shows significant inhibitory effects on the PI3K/ Akt pathway in osteoclasts and osteoblasts (Yuan et al. 2015; Hu ZC et al. 2019). However, little is known about the specific 
action of leonurine on PI3K/Akt in tumour cells. Therefore, to disclose the possible molecular mechanism underlying the anti-AML activity of leonurine, we studied the effects of leonurine on the PI3K/Akt pathway in HL-60 and U-937 cells. Our data showed that leonurine exhibited significant inhibitory effects on the phosphorylation of PI3K and Akt in HL-60 and U-937 cells. Previous reports (Lu et al. 2015; Yang et al. 2017) have proven the relationship of the PI3K/ Akt pathway with apoptosis. The PI3K inhibitor LY294002 has been proven to inhibit HL-60 proliferation and induce cell apoptosis. Moreover, the Akt inhibitor MK-2206 was reported to induce apoptosis in several different types of AML cells (MV-4-11, MOLM-13, OCI/AML3, and U-937). It is well accepted that some apoptotic death-related proteins are downstream of the PI3K/Akt pathway, such as caspase- 3 and Bad (Nepstad et al. 2020; Li et al. 2021). Therefore, our data suggest that inhibition of PI3K/Akt may be involved in the induction of the anti-AML action of leonurine. However, we cannot confirm the inhibitory effect of leonurine on the phosphorylation of PI3K and Akt in a direct or indirect manner, and more studies, such as molecular docking and other functional studies, will be needed to focus on the mutual integration between leonurine and PI3K/Akt.

Our present study revealed the anti-AML activity of leonurine both in vivo and in vitro. However, there were several limitations, which need to be improved and investigated in our next study. First, more AML models in vivo should be established to evaluate the anti-AML action of leonurine, including transplanted tumour models induced by AML cell lines with different biological characteristics and patientderived cell xenotransplant models. Second, many anti-AML cytotoxic drugs cause severe side effects, such as cardiac and gastrointestinal toxicity (Liso et al. 1990). Leonurine showed good protective effects on cytotoxic drug-induced tissue toxicity or cytotoxicity (Xin et al. 2009; Liu et al. 2018). Although we have found that leonurine together with MTX or vincristine synergistically inhibits HL-60 cell viability in vitro, more research is needed to evaluate potential benefits based on the therapeutic effect as well as side effects when using cytotoxic drugs and leonurine together. Finally, we have observed PBMCs from healthy donor are insensitive to leonurine treatment. However, PBMCs with different "status",
A

\section{Control

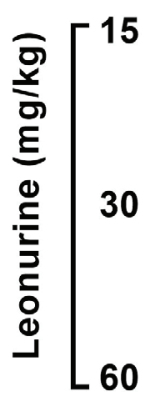

B
HL-60
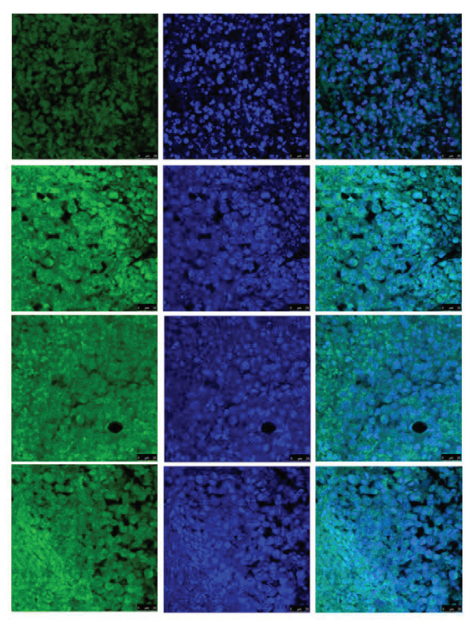

U-937
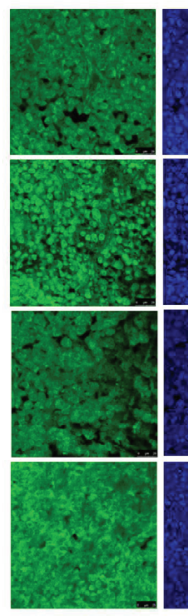

\section{U-937}

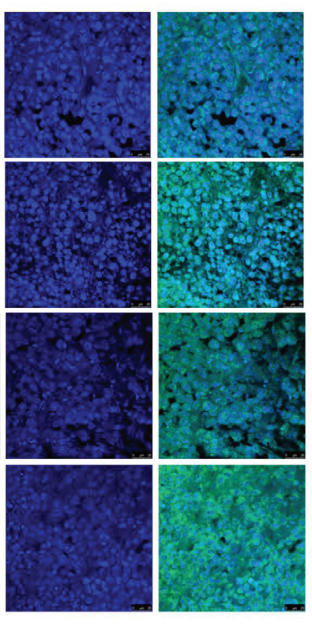

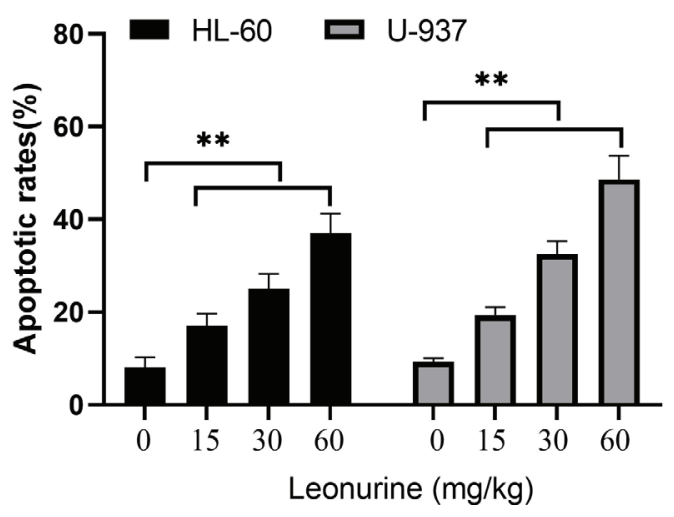

Figure 7. Leonurine promotes apoptosis of HL-60 and U-937 xenografted nude mice. At the end of the study, the tumour tissue was removed and labelled with TUNEL dye (A), and the apoptosis rates were calculated by counting at least 100 cells in three random fields $(\mathbf{B})$. Data are presented as the mean $\pm \mathrm{SD} ; n=6,{ }^{* *} p<0.01$ compared with the Control group (0 mg/kg leonurine). 
such as from healthy individuals or individuals with AML, may exhibit different responses to leonurine treatment.

In summary, we demonstrated that leonurine treatment could induce apoptosis of HL-60 and U-937 cells, resulting in the inhibition of cell proliferation in vitro and growth in vivo. Leonurine-induced cell death in AML cells involves the activation of intrinsic and extrinsic apoptotic pathways and the repression of PI3K and Akt phosphorylation.

Conflicts of interest. The authors declare that they have no competing interests.

\section{References}

Bertacchini J, Heidari N, Mediani L, Capitani S, Shahjahani M, Ahmadzadeh A, Saki N (2015): Targeting PI3K/AKT/mTOR network for treatment of leukaemia. Cell. Mol. Life. Sci. 72, 2337-2347 https://doi.org/10.1007/s00018-015-1867-5

Hirano T, Oka K, Mimaki Y, Kuroda M, Sashida Y (1996): Potent growth inhibitory activity of a novel Ornithogalum cholestane glycoside on human cells: induction of apoptosis in promyelocytic leukaemia HL-60 cells. Life Sci. 58, 789-798 https://doi.org/10.1016/0024-3205(95)02357-7

Hu PF, Sun FF, Qian J (2019): Leonurine exerts anti-catabolic and anti-apoptotic effects via nuclear factor kappa B (NF- $\mathrm{B})$ and mitogen-activated protein kinase (MAPK) signalling pathways in chondrocytes. Med. Sci. Monit. 25, 6271-6280 https://doi.org/10.12659/MSM.916039

Hu ZC, Gong LF, Li XB, Fu X, Xuan JW, Feng ZH, Ni WF (2019): Inhibition of $\mathrm{PI} 3 \mathrm{~K} / \mathrm{Akt} / \mathrm{NF}-\mathrm{kB}$ signalling with leonurine for ameliorating the progression of osteoarthritis: In vitro and in vivo studies. J. Cell. Physiol. 234, 6940-6950 https://doi.org/10.1002/jcp.27437

Juliusson G, Abrahamsson J, Lazarevic V, Antunovic P, Derolf Å, Garelius H, Lehmann S, Myhr-Eriksson K, Möllgård L, Uggla B, et al. (2017): Swedish AML Group and the Swedish Childhood Leukaemia Group. Prevalence and characteristics of survivors from acute myeloid leukaemia in Sweden. Leukaemia 31, 728-731 https://doi.org/10.1038/leu.2016.312

Kadia TM, Ravandi F, Cortes J, Kantarjian H (2016): New drugs in acute myeloid leukaemia. Ann. Oncol. 27, 770-778 https://doi.org/10.1093/annonc/mdw015

Kuykendall A, Duployez N, Boissel N, Lancet JE, Welch JS (2018): Acute myeloid leukaemia: the good, the bad, and the ugly. Am. Soc. Clin. Oncol. Educ. Book. 38, 555-573 https://doi.org/10.1200/EDBK_199519

Li B, Wu J, Li X (2013): Simultaneous determination and pharmacokinetic study of stachydrine and leonurine in rat plasma after oral administration of Herba Leonuri extract by LC-MS/MS. J. Pharm. Biomed. Anal. 76, 192-199 https://doi.org/10.1016/j.jpba.2012.12.029

Li N, Xu Q, Liu Q, Pan D, Jiang Y, Liu M, Liu M, Xu H, Lin C (2017): Leonurine attenuates fibroblast-like synoviocyte-mediated synovial inflammation and joint destruction in rheumatoid arthritis. Rheumatology (Oxford) 56, 1417-1427 https://doi.org/10.1093/rheumatology/kex142

Li ZL, Mi J, Lu L, Luo Q, Liu X, Yan YM, Jin B, Cao YL, Zeng XX, Ran LW (2021): The main anthocyanin monomer of Lycium ruthenicum Murray induces apoptosis through the ROS/PTEN/ $\mathrm{PI} 3 \mathrm{~K} / \mathrm{Akt} /$ caspase 3 signalling pathway in prostate cancer DU145 cells. Food Funct. 12, 1818-1828 https://doi.org/10.1039/D0FO02382E

Liso V, Specchia G, Pavone V, Capalbo S, Dione R (1990): Continuous infusion chemotherapy with epirubicin and vincristine in relapsed and refractory acute leukaemia. Acta Haematol. 83, 116-119 https://doi.org/10.1159/000205185

Liu C, Guo W, Maerz S, Gu X, Zhu Y (2013): 3,5-Dimethoxy4-(3-(2-carbonyl- ethyldisulfanyl)-propionyl)-benzoic acid 4-guanidino-butyl ester: a novel twin drug that prevents primary cardiac myocytes from hypoxia-induced apoptosis. Eur. J. Pharmacol. 700, 118-126 https://doi.org/10.1016/j.ejphar.2012.11.028

Liu X, Cao W, Qi J, Li Q, Zhao M, Chen Z, Zhu J, Huang Z, Wu L, Zhang B, et al. (2018): Leonurine ameliorates adriamycininduced podocyte injury via suppression of oxidative stress. Free Radic. Res. 52, 952-960 https://doi.org/10.1080/10715762.2018.1500021

Liu ZL, Zhu WR, Zhou WC, Ying HF, Zheng L, Guo YB, Chen JX, Shen XH (2014): Traditional Chinese medicinal herbs combined with epidermal growth factor receptor tyrosine kinase inhibitor for advanced non-small-cell lung cancer: a systematic review and meta-analysis. J. Integr. Med. 12, 346-358 https://doi.org/10.1016/S2095-4964(14)60034-0

Lu JW, Lin YM, Lai YL, Chen CY, Hu CY, Tien HF, Ou DL, Lin LI (2015): MK-2206 induces apoptosis of AML cells and enhances the cytotoxicity of cytarabine. Med. Oncol. 32, 206 https://doi.org/10.1007/s12032-015-0650-7

Mao F, Zhang L, Cai MH, Guo H, Yuan HH (2015): Leonurine hydrochloride induces apoptosis of H292 lung cancer cell by a mitochondria-dependent pathway. Pharm. Biol. 53, 1684-1690

https://doi.org/10.3109/13880209.2014.1001406

Ma Y, Zhu B, Yong L, Song C, Liu X, Yu H, Wang P, Liu Z, Liu X (2016): Regulation of intrinsic and extrinsic apoptotic pathways in osteosarcoma cells following oleandrin treatment. Int. J. Mol. Sci. 17, E1950 https://doi.org/10.3390/ijms17111950

Mans DR, da Rocha AB, Schwartsmann G (2000): Anticancer drug discovery and development in Brazil: targeted plant collection as a rational strategy to acquire candidate anticancer compounds. Oncologist. 5, 185-198 https://doi.org/10.1634/theoncologist.5-3-185

Nguyen LX, Sesay A, Mitchell BS (2014): Effect of CAL-101, a PI3K $\delta$ inhibitor, on ribosomal rna synthesis and cell proliferation in acute myeloid leukaemia cells. Blood Cancer J. 4, e228 https://doi.org/10.1038/bcj.2014.49

Nepstad I, Hatfield KJ, Grønningsæter IS, Reikvam H (2020): The PI3K-Akt-mTOR signalling pathway in human acute myeloid leukaemia (AML) cells. Int. J. Mol. Sci. 21, 2907 https://doi.org/10.3390/ijms21082907

Qi F, Zhao L, Zhou A, Zhang B, Li A, Wang Z, Han J (2015): The advantages of using traditional Chinese medicine as an adjunc- 
tive therapy in the whole course of cancer treatment instead of only terminal stage of cancer. Biosci. Trends 9, 16-34 https://doi.org/10.5582/bst.2015.01019

Riedhammer C, Halbritter D, Weissert R (2016): Peripheral blood mononuclear cells: isolation, freezing, thawing, and culture. Methods. Mol. Biol. 1304, 53-61 https://doi.org/10.1007/7651_2014_99

Rosen DB, Harrington KH, Cordeiro JA, Leung LY, Putta S, Lacayo N, Laszlo GS, Gudgeon CJ, Hogge DE, Hawtin RE, et al. (2013): AKT signalling as a novel factor associated with in vitro resistance of human AML to gemtuzumab ozogamicin. PLos One 8, e53518 https://doi.org/10.1371/journal.pone.0053518

Shi XR, Hong ZY, Liu HR, Zhang YC, Zhu YZ (2011): Neuroprotective effects of SCM198 on 6-hydroxydopamine-induced behavioural deficit in rats and cytotoxicity in neuronal $\mathrm{SH}$ SY5Y cells. Neurochem. Int. 58, 851-860 https://doi.org/10.1016/j.neuint.2010.11.007

Tamburini J, Elie C, Bardet V, Chapuis N, Park S, Broët P, CornilletLefebvre P, Lioure B, Ugo V, Blanchet O, et al. (2007): Constitutive phosphoinositide 3-kinase/Akt activation represents a favourable prognostic factor in de novo acute myelogenous leukaemia patients. Blood 110, 1025-1028 https://doi.org/10.1182/blood-2006-12-061283

Thomas DA, Sarris AH, Cortes J, Faderl S, O'Brien S, Giles FJ, Garcia-Manero G, Rodriguez MA, Cabanillas F, Kantarjianet $\mathrm{H}$ (2006): Phase II study of sphingosomal vincristine in patients with recurrent or refractory adult acute lymphocytic leukaemia. Cancer 106, 120-127 https://doi.org/10.1002/cncr.21595

Williamson SK, Johnson GA, Maulhardt HA, Moore KM, McMeekin DS, Schulz TK, Reed GA, Roby KF, Mackay CB, Smith HJ, et al. (2015): A phase I study of intraperitoneal nanoparticulate paclitaxel $\left(\operatorname{Nanotax}^{\oplus}\right)$ in patients with peritoneal malignancies. Cancer Chemother. Pharmacol. 75, 1075-1087 https://doi.org/10.1007/s00280-015-2737-4

Wu H, Dai A, Chen X, Yang X, Li X, Huang C, Jiang K, Deng G (2018): Leonurine ameliorates the inflammatory responses in lipopolysaccharide-induced endometritis. Int. Immunopharmacol. 61, 156-161 https://doi.org/10.1016/j.intimp.2018.06.002
Xie Y, Shi X, Sheng K, Han G, Li W, Zhao Q, Jiang B, Feng J, Li J, Gu Y (2019): PI3K/Akt signalling transduction pathway, erythropoiesis and glycolysis in hypoxia (Review). Mol. Med. Rep. 19, 783-791

https://doi.org/10.3892/mmr.2018.9713

Xin H, Liu XH, Zhu YZ (2009): Herba leonurine attenuates doxorubicin-induced apoptosis in H9c2 cardiac muscle cells. Eur. J. Pharmacol. 612, 75-79 https://doi.org/10.1016/j.ejphar.2009.03.067

Xu L, Jiang X, Wei F, Zhu H (2018): Leonurine protects cardiac function following acute myocardial infarction through anti-apoptosis by the PI3K/AKT/GSK3 $\beta$ signalling pathway. Med. Rep. 18, 1582-1590 https://doi.org/10.3892/mmr.2018.9084

Xu Q, Simpson SE, Scialla TJ, Bagg A, Carroll M (2003): Survival of acute myeloid leukaemia cells requires PI3 kinase activation. Blood 102, 972-980 https://doi.org/10.1182/blood-2002-11-3429

Yang H, Huang Y, Zou Y, Ma X (2017): Synergistic effects of phenylhexyl isothiocyanate and LY294002 on the PI3K/Akt signalling pathway in HL-60 cells. Oncol. Lett. 14, 3043-3050 https://doi.org/10.3892/ol.2017.6556

Yuan FL, Xu RS, Jiang DL, He XL, Su Q, Jin C, Li X. (2015): Leonurine hydrochloride inhibits osteoclastogenesis and prevents osteoporosis associated with oestrogen deficiency by inhibiting the NF- $\mathrm{kB}$ and PI3K/Akt signalling pathways. Bone 75, 128-137 https://doi.org/10.1016/j.bone.2015.02.017

Yu SJ, Cho IA, Kang KR, Jung YR, Cho SS, Yoon G, Oh JS, You JS, Seo YS, Lee GJ, et al. (2017): Licochalcone-E induces caspasedependent death of human pharyngeal squamous carcinoma cells through the extrinsic and intrinsic apoptotic signalling pathways. Oncol. Lett. 13, 3662-3668 https://doi.org/10.3892/ol.2017.5865

Zhu YZ, Wu W, Zhu Q, Liu X (2018): Discovery of Leonuri and therapeutical applications: From bench to bedside. Pharmacol. Ther. 188, 26-35 https://doi.org/10.1016/j.pharmthera.2018.01.006

Received: January 16, 2021

Final version accepted: May 26, 2021 